\title{
Paramètres d'évaluation des programmes de prévention des blessures chez les jeunes travailleurs occupant des professions à haut risque : examen de la portée de la littérature
}

\author{
Jennifer Smith, BFA (1,2); Birinder Praneet Purewal, D.M.D., BDS, M. Sc. (1); Alison Macpherson, Ph. D. (3); \\ Ian Pike, Ph. D. $(1,2,4)$
}

Cette synthèse des données probantes a fait l'objet d'une évaluation par les pairs.

\section{Résumé}

Introduction. Malgré la protection juridique dont les jeunes travailleurs au Canada font l'objet, les jeunes de 15 à 24 ans sont exposés à un risque élevé de blessures traumatiques au travail. Étant donné que de nombreuses initiatives de prévention des blessures ciblant les jeunes travailleurs existent, les défenseurs des droits des jeunes et les employeurs doivent relever le défi de choisir quels aspects de la prévention vont être les plus efficaces afin d'y consacrer leurs efforts. Une analyse de la littérature universitaire et de la littérature grise a été entreprise afin de compiler les paramètres (indicateurs évalués et méthodes de mesure) couramment utilisés pour évaluer les programmes de prévention des blessures auprès des jeunes travailleurs. Les paramètres constituent des références de mesure permettant d'évaluer l'efficacité, le rendement, le progrès ou la qualité d'un projet, d'un procédé ou d'un produit.

Méthodologie. Nous avons utilisé le modèle PICO (population, intervention, comparaison, résultat) pour définir les termes de recherche. Des recherches ont été effectuées dans Medline, PubMed, OVID, EMBASE, CCOHS, PsychINFO, CINAHL, NIOSHTIC, Google Scholar et dans la littérature grise afin de trouver des articles en anglais publiés entre 1975 et 2015. Deux lecteurs critiques indépendants ont examiné la liste des articles et ont classé les paramètres en trois catégories de prévention des blessures : l'éducation, l'environnement et l'application de règlements.

Résultats. Sur les 174 articles ayant répondu aux critères d'inclusion, 21 décrivaient et évaluaient une intervention. Parmi ceux-ci, la moitié $(n=11)$ étaient à caractère éducatif. Les paramètres couramment évalués étaient : les connaissances, les perceptions, les comportements ou les intentions autodéclarées, l'exposition aux dangers, les demandes d'indemnisation pour blessures et le taux d'accidents de travail. Une étude présentait une méthode destinée à élaborer des paramètres permettant de prédire les taux de blessure.

Conclusion. Des paramètres spécifiques à l'évaluation des programmes de prévention des blessures chez les jeunes travailleurs sont nécessaires, car les paramètres actuels sont insuffisants pour prévoir la diminution du nombre de blessures en lien avec la mise en place de programmes. De notre analyse ressort une étude apte à constituer un modèle pertinent pour les recherches futures visant à élaborer des paramètres de base valides destinés aux jeunes travailleurs, afin de les appliquer ensuite aux programmes de prévention des blessures chez les jeunes.

Mots-clés : jeunes travailleurs, accidents de travail, indicateurs de blessure, santé et sécurité au travail
Points saillants

- Les jeunes travailleurs de 15 à 24 ans présentent un risque de blessure plus élevé que les autres groupes d'âge et ont besoin de protections supplémentaires pour contrer les facteurs sociaux, développementaux, culturels et environnementaux qui contribuent à leur vulnérabilité.

- Les initiatives de sécurité en milieu de travail destinées aux jeunes travailleurs peuvent être classées en trois catégories de prévention des blessures : l'éducation, l'environnement et l'application de règlements.

- Des paramètres permettant de prévoir les blessures avant qu'elles ne se produisent sont nécessaires afin d'évaluer correctement l'efficacité des programmes de prévention des blessures.

- Cette analyse regroupe les paramètres couramment utilisés pour évaluer les programmes de prévention des blessures chez les jeunes travailleurs et fait ressortir la nécessité de mettre en place des paramètres plus fiables spécifiques aux jeunes travailleurs afin de créer des programmes fondés sur des données probantes.

\section{Rattachement des auteurs :}

1. British Columbia Injury Research and Prevention Unit, Vancouver (Colombie-Britannique), Canada

2. British Columbia Children's Hospital Research Institute, Vancouver (Colombie-Britannique), Canada

3. Faculté de la santé, École de kinésiologie et des sciences de la santé, Université York, Toronto (Ontario), Canada

4. Département de pédiatrie, Université de la Colombie-Britannique, Vancouver (Colombie-Britannique), Canada

Correspondance : Jennifer Smith, F508, 4480, rue Oak, Vancouver (Colombie-Britannique) V6H 3V4; tél. : 604-875-2000, poste 5478; courriel : jsmith@bcchr.ca 


\section{Introduction}

Les jeunes de 15 à 24 ans constituent environ $14 \%$ de la population active au Canada $^{1}$. Plus de 2,4 millions de jeunes, soit $54 \%$ de leur groupe d’âge, déclarent avoir un emploi chaque année $e^{2,3}$. De plus, les jeunes travailleurs présentent un risque d'accident de travail plus élevé que les groupes plus âgés ${ }^{4}$. Entre 2011 et 2013, près de 93000 jeunes Canadiens ont subi un accident de travail nécessitant un arrêt de travail ${ }^{5}$. De nombreux États reconnaissent la nécessité de prendre des mesures spéciales afin de protéger les jeunes travailleurs et réglementent donc les conditions de travail des jeunes. Le gouvernement du Canada considère que certains types de travail sont intrinsèquement "à risque élevé ", c'est-à-dire qu'ils sont susceptibles d'être en quelque sorte préjudiciables à la santé, à la sécurité ou au développement d'un jeune. Les jeunes de moins de 17 ans ne peuvent donc pas exercer ces professions, mais ceux âgés de 17 ans et plus peuvent travailler dans des milieux à risque élevé si les lois provinciales ou territoriales le permettent ${ }^{6,7}$. Malgré cette protection juridique, une étude récente a révélé que les jeunes travailleurs de moins de 17 ans subissaient des blessures graves telles que des brûlures, des blessures aux yeux, des blessures par écrasement, des amputations et des électrocutions à un taux plus élevé que leurs pairs sans emploi ${ }^{8}$. En outre, bon nombre de ces blessures sont survenues dans le secteur du commerce et dans les secteurs primaire et tertiaire ${ }^{8}$. Les taux élevés de blessures subies par les jeunes travailleurs sont préoccupants en matière de santé et de sécurité, en particulier dans les professions à risque élevé.

Les travailleurs de 15 à 24 ans sont vulnérables aux accidents de travail, en partie parce qu'ils n'ont pas d'expérience'. Le simple fait d'être nouveau sur un lieu de travail peut constituer une menace à la santé et à la sécurité du travailleur, car les travailleurs sans expérience ne connaissent pas l'environnement et ne possèdent habituellement pas de formation professionnelle suffisante ${ }^{10}$. Beaucoup de jeunes ne connaissent pas leurs droits et sont donc mal outillés pour demander une formation afin d'identifier et de gérer correctement les dangers éventuels ${ }^{10}$. Les jeunes peuvent aussi se sentir intimidés au travail et sentir qu'ils n'ont aucun pouvoir sur leurs conditions de travail ou peuvent être trop timides pour exprimer leurs préoccupations ${ }^{11}$.
D’autres facteurs communs associés au rôle des jeunes travailleurs dans la culture $\mathrm{du}$ milieu de travail exposent ces travailleurs à un risque plus élevé de blessures. Les jeunes travailleurs occupent souvent des emplois à temps partiel, saisonniers ou temporaires, ce qui peut mener à une fragmentation de la culture de sécurité et à des lacunes dans la formation. De telles circonstances influencent les perceptions qu'ont les jeunes travailleurs de la santé au travail et de sa pertinence pour eux. Beaucoup d'entre eux se disent que " cela fait partie du travail » et considèrent les blessures mineures telles que les brûlures, les coupures ou les égratignures comme normales et acceptables puisqu'elles se produisent régulièrement et qu'elles ne sont pas graves $^{12}$. En outre, les jeunes travailleurs peuvent décider de ne pas signaler une blessure par peur de provoquer une réaction négative de la part des autres et de compromettre leur emploi ${ }^{12}$. La vulnérabilité spécifique des jeunes travailleurs constitue un problème à plusieurs dimensions : sociale, culturelle, environnementale et liée au développement.

Étant donné qu'il existe de nombreuses initiatives de prévention des blessures ciblant les jeunes travailleurs, les défenseurs des droits des jeunes et les employeurs doivent relever le défi de choisir quels aspects de prévention vont être les plus efficaces afin d'y consacrer leurs efforts. Les initiatives de prévention des blessures peuvent habituellement être classées en trois catégories : l'environnement, l'application de règlements et l'éducation. La modification de l'environnement peut protéger les jeunes travailleurs en éliminant les dangers physiques, alors que l'application de politiques relatives à la sécurité peut favoriser une culture de protection au travail. La troisième catégorie, l'éducation, est l'approche de prévention des blessures la plus populaire, car elle est rentable, elle peut être mise en œuvre rapidement et sa portée est très large. Cependant, l'éducation n'est souvent pas suffisante pour générer des changements de comportement : d'autres obstacles et facteurs de changements doivent être examinés dans les deux autres catégories. La meilleure approche est probablement une combinaison d'efforts en matière d'éducation, d'environnement et d'application de règlements, de telle sorte que chaque catégorie renforce et soutienne les autres ${ }^{13}$.

Les avantages d'un programme de sécurité bien conçu sont largement corroborés par l'absence de blessures, ce qui est difficile à mesurer à l'avance. Dans chaque sphère de prévention des blessures, il est préférable d'élaborer des paramètres aptes à prédire les blessures avant que celles-ci ne se produisent, rendant ainsi possible l'évaluation d'une intervention sans devoir attendre une blessure. Cet examen porte ainsi sur les questions de santé et de sécurité au travail propres aux jeunes travailleurs et on y décrit et classe les paramètres couramment utilisés pour évaluer les initiatives de prévention des blessures chez les jeunes. L'efficacité de la prévention des blessures augmente lorsque les programmes visant les jeunes travailleurs sont mesurés et évalués correctement. Par ailleurs, l'harmonisation des normes de santé et de sécurité au travail entre les différentes instances est facilitée lorsque des paramètres communs sont adoptés à grande échelle.

Les objectifs spécifiques de cet examen de la portée de la littérature sont :

- identifier les paramètres actuellement en usage pour mesurer l'effet des programmes de prévention des blessures chez les jeunes travailleurs et des initiatives de sécurité au travail;

- classer ces paramètres en trois catégories de prévention des blessures couramment utilisées : l'éducation, l'environnement et l'application de règlements ${ }^{13}$;

- résumer les principales considérations émergeant de chaque catégorie et identifier les lacunes pour des recherches futures.

\section{Méthodologie}

\section{Critères d'inclusion}

Nous avons pris en compte les études évaluées par les pairs ainsi que les rapports et autres articles, publiés ou non, si : 1) les jeunes travailleurs de moins de 25 ans étaient inclus dans la population étudiée, 2) étaient abordées certaines caractéristiques des accidents de travail, et 3) ils s'appliquaient aux lieux de travail réglementés par le gouvernement fédéral du Canada ${ }^{7}$.

\section{Stratégie de recherche}

L'équipe de recherche a élaboré des critères de recherche précis en collaboration avec deux bibliothécaires universitaires. Cette 
recherche a utilisé notamment des termes précis décrivant les blessures ou les décès au travail chez les jeunes. La stratégie de recherche finale est présentée dans le tableau 1. Un lecteur critique a cherché des articles en anglais publiés entre 1975 et 2015 dans les bases de données suivantes : Medline, PubMed, OVID, EMBASE, CCOHS, PsychINFO, CINAHL, NIOSHTIC et Google Scholar.

\section{Identification et sélection des études}

La liste finale a été soumise à trois étapes de sélection. Lors de la première étape, les titres et les résumés des articles ont été examinés pour s'assurer que les articles respectaient les critères d'inclusion. Si les critères n'étaient pas visibles dans le titre ou le résumé, la partie "méthodologie » était analysée. Lors de la deuxième étape, deux lecteurs critiques indépendants ont examiné et classé les articles en fonction du thème principal de chaque article : " éducation », " environnement ", " application de règlements » ou " général » (pour ceux qui ne correspondaient pas spécifiquement à l'une des trois premières catégories). Les doublons ont été éliminés et les lecteurs ont discuté de leurs divergences jusqu'à l'atteinte d'un consensus. À la dernière étape, les articles ont été examinés en profondeur et classés en catégories selon s'ils traitaient de l'épidémiologie des blessures des travailleurs, de certains aspects de l'évaluation des risques ou encore d'une intervention.

\section{Abstraction et analyse de données}

Les données des articles ont été transcrites dans un tableau récapitulatif et les paramètres ont été extraits en fonction de

ce qui était mesuré (résultats) et de la manière dont la mesure était prise (méthodologie). Dans les cas où un paramètre répondait aux deux critères, on a considéré la source des données comme la méthode. Par exemple, pour le paramètre " nombre de décès dus à une blessure " répondant aux deux critères, le " décès " a été considéré comme le résultat et les " rapports du coroner " ou les "dossiers d'hospitalisation " comme la méthodologie. Nous avons sélectionné les articles décrivant et évaluant dans le même temps une intervention afin d'effectuer une analyse thématique en fonction de la pertinence du contexte professionnel, des principaux résultats, des points saillants de la section d'analyse, des limites ou recommandations de chaque étude, et nous avons fait de même pour les articles appartenant à chacune des trois catégories.

\section{Résultats}

Nous avons sélectionné 174 articles sur les accidents de travail s'appliquant aux jeunes travailleurs. La figure 1 illustre le processus de classement des résultats. Globalement, $84 \%$ des articles visaient principalement à décrire les caractéristiques du travailleur et l'accident de travail. Beaucoup d'entre eux portaient sur des blessures survenues dans les milieux agricoles, manufacturiers ou de la santé.

Vingt et un articles décrivaient et évaluaient une intervention applicable aux jeunes travailleurs, dont 11 (52 \%) portant sur une intervention éducative (tableau 2). Nombreux sont ceux qui ont utilisé des effets indirects pour mesurer l'efficacité, par exemple des modifications dans les connaissances, des risques perçus, des intentions,

TABLEAU 1

Termes de recherche dans le cadre PICO final

\begin{tabular}{|c|c|c|c|c|}
\hline $\mathbf{P}=$ Blessure & I = Intervention & C1 = Âge & $\mathrm{C} 2$ = Lieu de travail & $\mathbf{0}=$ Résultat \\
\hline injury & data collection & young adult & workplace & guideline \\
\hline $\begin{array}{l}\text { "wounds and } \\
\text { injuries" }\end{array}$ & focus groups & adolescent & workplace ${ }^{*}$ & legislation \\
\hline wound ${ }^{*}$ & $\begin{array}{l}\text { health impact } \\
\text { assessment }\end{array}$ & student & jobsite & law $^{*}$ \\
\hline injur* & health surveys & $\begin{array}{l}\text { vocational } \\
\text { education }\end{array}$ & & policy \\
\hline hazard* & $\begin{array}{l}\text { health status } \\
\text { indicators }\end{array}$ & young adj2 adult ${ }^{*}$ & & polic ${ }^{*}$ \\
\hline accident* & standards [st.fs.] & teen & & program* \\
\hline exposure* & & adolescen* & & \\
\hline
\end{tabular}

Abréviation : PICO, population, intervention, comparaison, résultat. des comportements et de la qualité du programme. Ces effets sont considérés comme indirects car ils ne mènent pas nécessairement à un nombre moins élevé de blessures ou à des blessures moins graves. Deux études ont mesuré directement la diminution du nombre de blessures, une étude a mesuré la réduction des coûts et une autre la diminution de l'exposition aux dangers $^{14-16}$.

Cinq études (24\%) portaient sur les effets d'une intervention environnementale (tableau 3) : trois avaient mesuré les effets des expositions aux dangers ${ }^{17-19}$ et les deux autres avaient évalué l'effet sur le comportement, les intentions et l'efficacité perçue $^{20-21}$.

Cinq études (24\%) portaient sur une intervention visant à faire appliquer des politiques ou des mesures de sécurité (tableau 4). Deux d'entre elles avaient mesuré l'effet des interventions sur la diminution des blessures (incluant les blessures mortelles), l'une décrivant aussi les modifications législatives ayant découlé de l'intervention ${ }^{22-23}$. Deux autres avaient analysé l'effet des inspections de sécurité instaurées à la suite de l'intervention, et enfin la dernière avait mesuré les expositions aux dangers ${ }^{24-26}$

\section{Analyse}

Il existe un vaste corpus de recherches dans le domaine de la sécurité au travail chez les jeunes travailleurs décrivant la personne blessée ou décédée et les circonstances de l'accident. Un sous-ensemble important de ce corpus décrit les risques et les taux de blessures ainsi que leur gravité. Divers travaux tentent d'explorer les moyens d'éliminer ou de réduire les risques de blessures grâce à des interventions visant le travailleur sur le plan individuel ou collectif ou ciblant l'environnement ou la culture du lieu de travail. De nombreuses études décrivent ces interventions, mais peu les analysent, en particulier sous l'angle de leur effet sur la diminution des blessures.

\section{Éducation}

Il semble y avoir une abondance d'études décrivant des initiatives de prévention des blessures qui adoptent une approche éducationnelle, puisque l'évaluation du changement dans les connaissances semble être une tâche courante dans la société 
FIGURE 1

Schéma de la classification des articles et résumé des paramètres

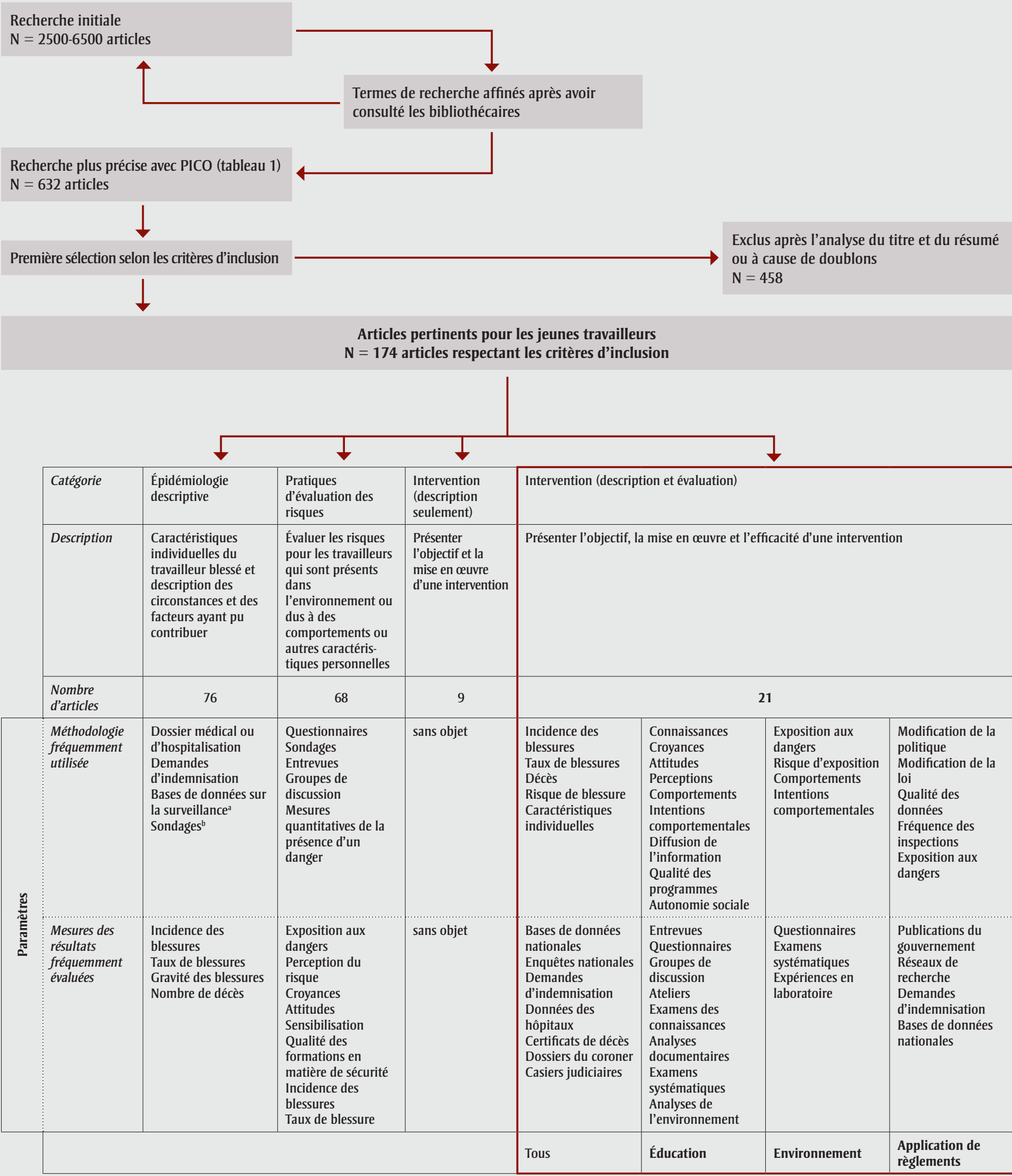

Abréviation : PICO, population, intervention, comparaison, résultat.

${ }^{a}$ Exemple : registres des traumatismes ou publications gouvernementales.

${ }^{\mathrm{b}}$ Exemple : National Health Interview Survey. 
TABLEAU 2

Études évaluant une intervention dans la catégorie de l'éducation

\begin{tabular}{|c|c|c|c|c|c|}
\hline Étude & Intervention & Contexte & Principales constatations & Mesures des résultats & Méthodologie \\
\hline $\begin{array}{l}\text { Arcury et } \\
\text { collab. }^{27}\end{array}$ & $\begin{array}{l}\text { Formation à la prévention } \\
\text { des accidents (programme } \\
\text { PACE) }\end{array}$ & $\begin{array}{l}\text { Agriculture } \\
\text { États-Unis }\end{array}$ & $\begin{array}{l}\text { - Le fait de recevoir de l'information sur la sécurité des } \\
\text { pesticides a diminué le risque perçu et a augomenté le } \\
\text { sentiment de contrôle } \\
\text { - Le risque perçu n'était pas lié au comportement } \\
\text { sécuritaire }\end{array}$ & $\begin{array}{l}\text { Effet sur les connaissances, le risque } \\
\text { perçu et le comportement }\end{array}$ & Entrevue \\
\hline $\begin{array}{l}\text { Banco et } \\
\text { collab. }^{15}\end{array}$ & $\begin{array}{l}\text { Formation à la prévention } \\
\text { des accidents et meilleurs } \\
\text { outils de coupe }\end{array}$ & $\begin{array}{l}\text { Commerce de } \\
\text { détail } \\
\text { États-Unis }\end{array}$ & $\begin{array}{l}\text { - Le nouvel outil de coupe combiné à une formation à la } \\
\text { prévention des accidents a été la façon la plus efficace } \\
\text { de réduire les coupures } \\
\text { - Le groupe ayant reçu seulement la formation avait un } \\
\text { taux de blessure similaire au groupe de contrôle }\end{array}$ & $\begin{array}{l}\text { Effet sur les connaissances, } \\
\text { l'incidence des accidents, les } \\
\text { demandes d'indemnisation et les } \\
\text { frais connexes }\end{array}$ & Expérimentation \\
\hline $\begin{array}{l}\text { Burke et } \\
\text { collab. }^{14}\end{array}$ & $\begin{array}{l}\text { Formation à la prévention } \\
\text { des accidents (variée) }\end{array}$ & $\begin{array}{l}\text { Général } \\
\text { Plusieurs pays }\end{array}$ & $\begin{array}{l}\text { À mesure que les exigences en matière de participa- } \\
\text { tion des apprenants augmentaient (engagement), } \\
\text { l'acquisition de connaissances augmentait et le taux } \\
\text { de blessures et de maladies diminuait } \\
\text { - Tous les niveaux de participation produisaient des } \\
\text { améliorations significatives du comportement }\end{array}$ & $\begin{array}{l}\text { Effet sur les connaissances, le } \\
\text { comportement et l'incidence des } \\
\text { accidents }\end{array}$ & $\begin{array}{l}\text { Examens } \\
\text { systématiques }\end{array}$ \\
\hline $\begin{array}{l}\text { Chin et } \\
\text { collab. }\end{array}$ & $\begin{array}{l}\text { Formation à la prévention } \\
\text { des accidents (variée) et } \\
\text { diffusion de renseigne- } \\
\text { ments }\end{array}$ & $\begin{array}{l}\text { Général } \\
\text { Canada }\end{array}$ & $\begin{array}{l}\text { - Les programmes de prévention des blessures chez les } \\
\text { jeunes travailleurs aux niveaux fédéral, provincial et } \\
\text { territorial contribuent peu à l'autonomie sociale } \\
\text { - Les programmes sont informatifs plutôt que } \\
\text { pédagogiques }\end{array}$ & $\begin{array}{l}\text { Effet sur le soutien de l'autonomie } \\
\text { sociale en quatre grandes } \\
\text { catégories: } \\
\text { 1. connaissance de soi } \\
\text { 2. connaissance de ses droits } \\
\text { 3. communication } \\
\text { 4. sens du leadership }\end{array}$ & $\begin{array}{l}\text { Analyse documen- } \\
\text { taire }\end{array}$ \\
\hline $\begin{array}{l}\text { Ehlers et } \\
\text { Graydon }^{33}\end{array}$ & $\begin{array}{l}\text { Formation à la prévention } \\
\text { des accidents et diffusion } \\
\text { de renseignements }\end{array}$ & $\begin{array}{l}\text { Agriculture } \\
\text { États-Unis }\end{array}$ & $\begin{array}{l}\text { Des partenariats avec des organisations importantes } \\
\text { de l'industrie permettent une large diffusion du } \\
\text { matériel pédagogique }\end{array}$ & $\begin{array}{l}\text { Effet sur l'engagement } \\
\text { communautaire }\end{array}$ & Atelier \\
\hline $\begin{array}{l}\text { Kahan et } \\
\text { collab. }^{28}\end{array}$ & $\begin{array}{l}\text { Formation à la prévention } \\
\text { des accidents (programme } \\
\text { de formation de } \\
\text { l'entreprise) }\end{array}$ & $\begin{array}{l}\text { Fabrication } \\
\text { Israël }\end{array}$ & $\begin{array}{l}\text { - La législation WRTK ne garantit pas que les } \\
\text { travailleurs sont au courant de leurs droits et des } \\
\text { dangers de l'emploi } \\
\text { - Le matériel de formation ne correspondait pas à la } \\
\text { langue des travailleurs ni à leur niveau } \\
\text { d'alphabétisation et d'éducation }\end{array}$ & Effet sur les connaissances & $\begin{array}{l}\text { Entrevue et } \\
\text { questionnaire }\end{array}$ \\
\hline $\begin{array}{l}\text { Linker et } \\
\text { collab. }^{34}\end{array}$ & $\begin{array}{l}\text { Formation à la prévention } \\
\text { des accidents (pro- } \\
\text { grammes des établisse- } \\
\text { ments d'enseignement } \\
\text { secondaire) }\end{array}$ & $\begin{array}{l}\text { Général } \\
\text { États-Unis }\end{array}$ & $\begin{array}{l}\text { - Les connaissances des étudiants ont augmenté après } \\
\text { avoir reçu l'intervention éducative }\end{array}$ & $\begin{array}{l}\text { Effet sur les connaissances et sur } \\
\text { l'évaluation de la facilité de la mise } \\
\text { en œuvre réalisée par le professeur }\end{array}$ & $\begin{array}{l}\text { Questionnaire et } \\
\text { évaluations avant et } \\
\text { après la formation }\end{array}$ \\
\hline $\begin{array}{l}\text { Schulte et } \\
\text { collab. }^{35}\end{array}$ & $\begin{array}{l}\text { Formation à la prévention } \\
\text { des accidents (pro- } \\
\text { grammes de préparation } \\
\text { au marché du travail) }\end{array}$ & $\begin{array}{l}\text { Général } \\
\text { États-Unis }\end{array}$ & $\begin{array}{l}\text { L'inclusion de renseignements sur la SST n'est pas } \\
\text { uniforme dans un très largé éventail de programmes } \\
\text { de préparation au marché du travail }\end{array}$ & $\begin{array}{l}\text { Présence ou non de renseignements } \\
\text { sur la SST dans le programme de } \\
\text { formation }\end{array}$ & $\begin{array}{l}\text { Analyse de l'environ- } \\
\text { nement }\end{array}$ \\
\hline $\begin{array}{l}\text { Teran et } \\
\text { collab. }^{36}\end{array}$ & $\begin{array}{l}\text { Formation à la prévention } \\
\text { des accidents (programme } \\
\text { ALS) }\end{array}$ & $\begin{array}{l}\text { Agriculture } \\
\text { États-Unis }\end{array}$ & $\begin{array}{l}\text { - Le programme d'ALS en milieu scolaire est un moyen } \\
\text { efficace de rejoindre les jeunes ouvriers agricoles } \\
\text { - Près de la moitié des membres du groupe } \\
\text { d'intervention ont déclaré mettre en œuvre les } \\
\text { comportements et } 73 \text { \% des membres ont déclaré } \\
\text { partager leurs nouvelles connaissances avec d'autres } \\
\text { - La participation des parents aux ateliers communau- } \\
\text { taires a été accueillie avec enthousiasme, mais n'a eu } \\
\text { aucun effet sur les résultats des étudiants }\end{array}$ & $\begin{array}{l}\text { Effet sur les connaissances, les } \\
\text { attitudes et le comportement }\end{array}$ & $\begin{array}{l}\text { Entrevue, groupes } \\
\text { de discussion et } \\
\text { évaluations avant et } \\
\text { après la formation }\end{array}$ \\
\hline $\begin{array}{l}\text { Lepping et } \\
\text { collab. }^{16}\end{array}$ & $\begin{array}{l}\text { Formation sur la gestion } \\
\text { de l'agressivité }\end{array}$ & $\begin{array}{l}\text { Hôpital } \\
\text { Royaume-Uni }\end{array}$ & $\begin{array}{l}\text { - Aucune corrélation entre la formation sur la gestion } \\
\text { de l'agressivité (formation sur la désescalade ou } \\
\text { formation sur la gestion de la violence) et une } \\
\text { fréquence moins élevée des expositions à la violence }\end{array}$ & $\begin{array}{l}\text { Effet sur la fréquence d'exposition } \\
\text { aux dangers (violence et agressivité) }\end{array}$ & $\begin{array}{l}\text { Questionnaire validé } \\
\text { (Survey of Violence } \\
\text { Experienced by } \\
\text { Staff) }\end{array}$ \\
\hline $\begin{array}{l}\text { Tucker et } \\
\text { Turner }^{37}\end{array}$ & $\begin{array}{l}\text { Formation à la prévention } \\
\text { des accidents (variée) et } \\
\text { campagnes de marketing } \\
\text { social (variées) }\end{array}$ & $\begin{array}{l}\text { Général } \\
\text { Canada }\end{array}$ & $\begin{array}{l}\text { Les jeunes travailleurs sont réticents à dénoncer un } \\
\text { travail dangereux parce que leurs croyances sur les } \\
\text { dangers de dénoncer persistent, malgré la } \\
\text { prédominance des campagnes de marketing social et } \\
\text { des programmes des écoles secondaires ciblées }\end{array}$ & $\begin{array}{l}\text { Effet sur les intentions de sécurité } \\
\text { (signaler une blessure ou un } \\
\text { problème de sécurité) }\end{array}$ & $\begin{array}{l}\text { Groupes de } \\
\text { discussion }\end{array}$ \\
\hline
\end{tabular}


TABLEAU 3

Études évaluant une intervention dans la catégorie de l'environnement

\begin{tabular}{|c|c|c|c|c|c|}
\hline Étude & Intervention & Contexte & Principales constatations & Mesures des résultats & Méthodologie \\
\hline $\begin{array}{l}\text { Adams et } \\
\text { collab. }^{20}\end{array}$ & $\begin{array}{l}\text { Signes avant- } \\
\text { coureurs }\end{array}$ & $\begin{array}{l}\text { Industrie lourde } \\
\text { Australie }\end{array}$ & $\begin{array}{l}\text { - L'effet de troisième personne : les } \\
\text { travailleurs évaluent que leurs propres } \\
\text { risques sont moins élevés que ceux des } \\
\text { autres employés } \\
\text { - Les panneaux sont jugés d'une } \\
\text { efficacité équivalente, même lorsqu'un } \\
\text { élément « essentiel » n'est pas présent }\end{array}$ & $\begin{array}{l}\text { Effet sur les intentions comportemen- } \\
\text { tales et sur l'efficacité perçue }\end{array}$ & Questionnaire \\
\hline $\begin{array}{l}\text { McDowell et } \\
\text { collab. }{ }^{17}\end{array}$ & EPI & $\begin{array}{l}\text { Nettoyage des } \\
\text { plages } \\
\text { États-Unis }\end{array}$ & $\begin{array}{l}\text { - Les gants anti-vibration ne sont pas } \\
\text { efficaces pour atténuer les } \\
\text { fréquences des vibrations et peuvent } \\
\text { même augómenter les vibrations } \\
\text { transmises et la fatigue ressentie } \\
\text { dans les mains et les bras }\end{array}$ & $\begin{array}{l}\text { Exposition au danger (vibration } \\
\text { transmise) et gravité du danger } \\
\text { (fréquence de vibration) }\end{array}$ & Appareil spécialisé \\
\hline $\begin{array}{l}\text { Salvatore et } \\
\text { collab. }^{21}\end{array}$ & $\begin{array}{l}\text { Stations/Postes de } \\
\text { lavage des mains, } \\
\text { EPI et séances } \\
\text { d'information } \\
\text { hebdomadaires }\end{array}$ & $\begin{array}{l}\text { Agriculture } \\
\text { États-Unis }\end{array}$ & $\begin{array}{l}\text { L'utilisation d'EPI et l'habitude de se } \\
\text { laver les mains se sont améliorées } \\
\text { pendant les heures de travail, mais } \\
\text { pas après le travail }\end{array}$ & Incidence sur le comportement & Questionnaire \\
\hline $\begin{array}{l}\text { Ulrey et } \\
\text { Fathallah }{ }^{18}\end{array}$ & $\begin{array}{l}\text { Dispositif de } \\
\text { transfert de poids } \\
\text { (BNDR) }\end{array}$ & $\begin{array}{l}\text { Industrie lourde } \\
\text { États-Unis }\end{array}$ & $\begin{array}{l}\text { Le dispositif réduit la douleur au bas } \\
\text { du dos pendant les tâches exécutées } \\
\text { avec une posture voûtée }\end{array}$ & $\begin{array}{l}\text { Effet sur l'activité musculaire et sur le } \\
\text { positionnement du corps }\end{array}$ & $\begin{array}{l}\text { Appareil spécialisé } \\
\text { (électromyographie) }\end{array}$ \\
\hline $\begin{array}{l}\text { Verbeek et } \\
\text { collab. }{ }^{19}\end{array}$ & $\begin{array}{l}\text { EPI et contrôles } \\
\text { techniques du } \\
\text { bruit }\end{array}$ & $\begin{array}{l}\text { Différents lieux } \\
\text { de travail } \\
\text { Plusieurs pays }\end{array}$ & $\begin{array}{l}\text { - La législation limitant l'exposition au } \\
\text { bruit a permis de réduire efficacement } \\
\text { l'exposition au danger dans une étude } \\
\text { - L'efficacité du port de bouchons } \\
\text { d'oreille dépend de la formation et de } \\
\text { leur bonne utilisation }\end{array}$ & $\begin{array}{l}\text { Effet sur le taux de blessures, sur } \\
\text { l'exposition au danger (bruit) et sur } \\
\text { l'incidence des blessures }\end{array}$ & $\begin{array}{l}\text { Revue systématique } \\
\text { (Cochrane) }\end{array}$ \\
\hline
\end{tabular}

Abréviations : BNDR, bending non-demand return; EPI, équipement de protection individuelle.

occidentale. Il est facile de comparer les résultats aux tests de connaissance obtenus avant et après un programme éducatif et des évaluations peuvent être réalisées périodiquement pour suivre l'évolution de la rétention des connaissances. En outre, les interventions éducationnelles sont populaires parce qu'elles peuvent être très flexibles en ce qui a trait à la portée, à l'application et au coût, ce qui en fait une option intéressante pour de nombreux employeurs. Du point de vue de la santé publique, l'éducation est une approche pratique puisque les programmes peuvent être intégrés à des programmes scolaires en cours, ciblant ainsi les travailleurs au moment de leur entrée sur le marché du travail. Les études présentées dans cette analyse montrent que de nombreux types d'interventions éducationnelles constituent une très bonne manière de produire le changement désiré sur le plan des connaissances.

Le lien entre les programmes d'éducation, les changements dans les connaissances et la prévention des blessures est moins évident. L'objectif final de l'éducation des travailleurs est d'obtenir des comportements spécifiques en matière de sécurité lorsque cela s'avère nécessaire. La mesure objective du comportement dans un contexte réel est difficile et peut s'avérer coûteuse, ce qui explique sans doute pourquoi on mesure habituellement les intentions comportementales ou les comportements autodéclarés en matière de sécurité. Ces mesures fournissent des renseignements utiles sur une partie de l'effet de l'éducation, mais ils ne permettent pas d'établir un lien direct avec une diminution du nombre de blessures.

Burke et ses collègues ont mené une métaanalyse d'études quasi expérimentales portant sur la relation entre la participation de l'apprenant et la diminution des blessures ${ }^{14}$. Ils ont conclu que tous les niveaux de participation dans les cours de sécurité menaient à une diminution des blessures, mais que les programmes d'étude demandant une participation plus importante entraînaient les diminutions les plus significatives ${ }^{14}$. Une autre étude expérimentale a évalué le rôle de l'éducation dans la diminution des coupures après que les travailleurs eurent reçu un outil de coupe supérieur sur le plan ergonomique ${ }^{15}$. Les demandes d'indemnisation des travailleurs ont été examinées pendant un an après l'adoption du nouvel outil. Les lieux de travail ayant reçu à la fois l'outil et une formation ont connu une diminution des réclamations plus forte $(-3,5$ blessures par 100000 heures-personnes) que les lieux ayant reçu seulement une formation $(-1,5)$ et que le groupe de contrôle $(-1,6)$. Les chercheurs ont conclu que la formation sur l'utilisation correcte du nouvel outil était un facteur déterminant dans la réduction du nombre de blessures ${ }^{15}$. Ces deux études ont montré que le nombre de blessures pouvait diminuer grâce à la formation, mais elles n’ont pas expliqué en détail comment la formation influence les blessures et pourquoi.

Il demeure qu'une légère éducation ou une courte formation vaut toujours mieux que rien. Enseigner aux jeunes travailleurs comment être en sécurité constitue donc une composante essentielle de tout programme de prévention des blessures. Les études présentées dans cette analyse suggèrent qu'au-delà de l'enrichissement des connaissances, il existe d'autres paramètres aptes à mesurer la qualité et l'effet de 
TABLEAU 4

Études évaluant une intervention dans la catégorie de l'application de règlements

\begin{tabular}{|c|c|c|c|c|c|}
\hline Étude & Intervention & Contexte & Principales constatations & Mesures des résultats & Méthodologie \\
\hline $\begin{array}{l}\text { Haviland et } \\
\text { collab. }^{23}\end{array}$ & $\begin{array}{l}\text { Inspections de } \\
\text { l'OSHA }\end{array}$ & $\begin{array}{l}\text { Fabrication } \\
\text { États-Unis }\end{array}$ & $\begin{array}{l}\text { - Les inspections avec pénalités ont eu un effet sur les } \\
\text { types de blessures liées ou non aux normes } \\
\text { - Les contraventions pour violation des exigences en } \\
\text { matière d'EPI ont eu l'effet le plus important sur la } \\
\text { prévention des blessures } \\
\text { - Les inspections peuvent inciter les gestionnaires à } \\
\text { prendre des mesures de sécurité qui vont au-delà de } \\
\text { la conformité aux normes }\end{array}$ & $\begin{array}{l}\text { Effet sur le taux de } \\
\text { blessures }\end{array}$ & $\begin{array}{l}\text { Dossiers d'inspection de } \\
\text { l'OSHA, dossiers du } \\
\text { State Department of } \\
\text { Labor and Industry, } \\
\text { dossiers de l'assurance- } \\
\text { emploi, demandes } \\
\text { d'indemnisation }\end{array}$ \\
\hline $\begin{array}{l}\text { Rowlinson et } \\
\text { Jia }^{26}\end{array}$ & $\begin{array}{l}\text { Protocole pour } \\
\text { une gestion du } \\
\text { stress thermique } \\
\text { au niveau des } \\
\text { travailleurs et de la } \\
\text { direction }\end{array}$ & $\begin{array}{l}\text { Travaux de } \\
\text { construction } \\
\text { Chine }\end{array}$ & $\begin{array}{l}\text { Un nouveau protocole pour l'élaboration de systèmes } \\
\text { de gestion du stress thermique pour les gestionnaires } \\
\text { qui décident des régimes d'alternance de travail et de } \\
\text { repos et de l'autodiscipline des travailleurs est plus } \\
\text { efficace que les lignes directrices actuelles }\end{array}$ & $\begin{array}{l}\text { Effet sur le stress } \\
\text { thermique et } \\
\text { déroulement du } \\
\text { travail }\end{array}$ & $\begin{array}{l}\text { Appareil spécifique } \\
\text { (température au } \\
\text { thermomètre-globe } \\
\text { mouillé) }\end{array}$ \\
\hline $\begin{array}{l}\text { Kica et } \\
\text { Rosenman }{ }^{25}\end{array}$ & $\begin{array}{l}\text { Amélioration de la } \\
\text { surveillance, ce } \\
\text { qui mène à des } \\
\text { inspections du lieu } \\
\text { de travail par la } \\
\text { MIOSHA }\end{array}$ & $\begin{array}{l}\text { Différents lieux } \\
\text { de travail } \\
\text { États-Unis }\end{array}$ & $\begin{array}{l}\text { - Le nouveau système de surveillance a permis } \\
\text { d'identifier un nombre beaucoup plus élevé de cas de } \\
\text { blessures qui ont conduit à des inspections sur le lieu } \\
\text { de travail } \\
\text { - Ces inspections n'auraient pas eu lieu autrement }\end{array}$ & $\begin{array}{l}\text { Effet sur le nombre } \\
\text { d'inspections des } \\
\text { lieux de travail }\end{array}$ & $\begin{array}{l}\text { Comparaison de cas } \\
\text { identifiés par le Bureau } \\
\text { of Labor Statistics et } \\
\text { nouvelles mesures de } \\
\text { surveillance (comprend } \\
\text { les dossiers hospitaliers } \\
\text { et les demandes } \\
\text { d'indemnisation) }\end{array}$ \\
\hline $\begin{array}{l}\text { Largo et } \\
\text { Rosenman }{ }^{24}\end{array}$ & $\begin{array}{l}\text { Amélioration de la } \\
\text { surveillance, ce } \\
\text { qui mène à des } \\
\text { inspections du lieu } \\
\text { de travail par la } \\
\text { MIOSHA }\end{array}$ & $\begin{array}{l}\text { Différents lieux } \\
\text { de travail } \\
\text { États-Unis }\end{array}$ & $\begin{array}{l}\text { - Le nouveau système de surveillance a permis } \\
\text { d'identifier un nombre beaucoup plus élevé de cas de } \\
\text { blessures qui ont conduit à des inspections sur le lieu } \\
\text { de travail } \\
\text { - Ces inspections n'auraient pas eu lieu autrement }\end{array}$ & $\begin{array}{l}\text { Effet sur le nombre } \\
\text { d'inspections des } \\
\text { lieux de travail }\end{array}$ & $\begin{array}{l}\text { Comparaison de cas } \\
\text { identifiés par le Bureau } \\
\text { of Labor Statistics et } \\
\text { nouvelles mesures de } \\
\text { surveillance (comprend } \\
\text { les dossiers hospitaliers } \\
\text { et les demandes } \\
\text { d'indemnisation) }\end{array}$ \\
\hline
\end{tabular}

Abréviations : EPI, équipement de protection individuelle; FACE, Fatality Assessment and Control Evaluation; MIOSHA, Michigan Occupational Safety and Health Administration; OSHA, Occupational Safety and Health Administration.

l'éducation : 1) le sentiment de contrôle à l'égard d'un risque spécifique, 2) l'adéquation entre le programme et le travailleur en ce qui concerne la langue, l'éducation, le niveau d'alphabétisation et le stade de développement et 3 ) le degré d'engagement du jeune travailleur dans le programme. Le premier paramètre a été présenté par Arcury et ses collègues pour induire un comportement plus sécuritaire chez les travailleurs $^{27}$. Les travailleurs ayant reçu une intervention éducationnelle ont acquis un plus grand sentiment de contrôle sur leur niveau personnel d'exposition aux risques, ce qui est directement lié à leur motivation à changer leur comportement ${ }^{27}$. Les deux autres paramètres sont associés à une acquisition importante de connaissances ${ }^{14,28}$. Bien qu'aucune étude n’ait évalué la rétention des connaissances à long terme, les études présentées dans cette analyse suggèrent que la valeur immédiate d'un programme d'éducation sera meilleure si les paramètres appropriés peuvent être identifiés et appliqués.

\section{Environnement}

Nous avons trouvé peu d'études analysant les interventions environnementales. Le fait d'apporter des modifications à l'environnement de travail ou de fournir des équipements de protection individuelle (EPI) nécessite des investissements considérables. Par ailleurs, deux études ont été menées dans un laboratoire en utilisant des appareils sophistiqués afin de prendre des mesures précises. Le coût associé à cette manière de produire des données est susceptible de constituer un obstacle important pour les chercheurs, ce qui pourrait expliquer le nombre limité d'études sur ce sujet.
Deux études avancent que le sentiment de sécurité peut interagir avec l'environnement pour augmenter la vulnérabilité des jeunes travailleurs. Burt et ses collègues ont constaté que les jeunes recrues avaient souvent des attentes en matière de sécurité qui ne correspondaient pas à la réalité du climat de sécurité de leur nouveau lieu de travail, ce qui augmentait les risques de blessures $^{29}$. Adams et ses collègues ont également montré un décalage entre le sentiment de sécurité et la réalité des dangers sur le lieu de travail ${ }^{20}$. Les chercheurs ont découvert que les panneaux d'avertissement de danger avaient un effet sur les intentions comportementales, mais qu'ils étaient également sujets à " l'effet de troisième personne ", ce qui veut dire que les travailleurs qui voyaient le panneau avaient tendance à penser que les autres étaient plus vulnérables qu'eux ${ }^{20}$. Ces études suggèrent qu'il pourrait être important de 
prendre en considération les paramètres servant à mesurer la perception du risque et la culture de sécurité lors de l'évaluation de l'environnement physique des jeunes travailleurs.

Davantage d'études sont nécessaires pour étudier l'effet précis des interventions environnementales sur les risques et le taux de blessures et sur leur gravité chez les jeunes travailleurs. Des études portant sur des facteurs autres que l'utilisation d'équipements de protection individuelle pour évaluer la conception de l'environnement physique de travail sont nécessaires. Ces moyens supplémentaires d'atténuer les risques et de protéger les travailleurs n'ont pas été examinés en profondeur dans les études présentées dans cette analyse.

\section{Application de règlements}

L'application de règlements a de vastes répercussions sur les travailleurs et les employeurs. Les politiques relatives à la sécurité ont une influence sur toutes les dimensions d'un milieu de travail. Les décideurs peuvent ainsi être amenés à devoir équilibrer la productivité ou l'efficacité et les exigences de sécurité, notamment lorsque l'une impose à l'autre des limites directes. L'élaboration, la mise en œuvre, la communication et l'application d'un changement de politique demandent beaucoup de temps et de travail. Si le changement est inefficace, le fait de l'annuler a des conséquences négatives énormes pour toutes les personnes concernées. Outre les dépenses occasionnées par l'intervention, des changements trop nombreux peuvent entraîner de la confusion à tous les niveaux d'une organisation et miner la confiance des travailleurs envers les gestionnaires, ce qui pourrait affecter le respect des règles de sécurité dans le futur. Les changements dans les politiques de sécurité (et à plus grande échelle, dans les lois) ont donc lieu à un rythme lent car ils nécessitent une consultation approfondie, une réelle participation des parties prenantes et des investissements pour s'assurer d'obtenir le résultat désiré.

Du fait de ces considérations, très peu d'études analysant l'application de politiques de sécurité ont été trouvées. De plus, les études de cette catégorie étaient majoritairement qualitatives : elles contenaient des descriptions très détaillées de la façon d'obtenir des changements significatifs de politiques ou de lois et présentaient les effets de ces changements sur les taux de blessures et les accidents de travail mortels. Il convient de souligner que deux des cinq études avaient analysé le rôle que joue la surveillance de la qualité dans la diminution du nombre de blessures ${ }^{24-25}$. Les chercheurs ont montré que lors de la surveillance de la qualité, on devrait non seulement obtenir des données provenant de sources multiples, mais aussi se connecter à un réseau pour exploiter les données en temps opportun. Les inspections de sécurité avaient été instaurées plus rapidement et dans un plus grand nombre de cas car des contacts de l'Occupational Safety and Health Administration (OSHA) étaient membres du réseau de surveillance. Il est évident qu'une collaboration est nécessaire dans un cadre de mise en application de règlements pour s'assurer que des données utiles sont recueillies puis efficacement exploitées. Les chercheurs ont également montré qu'on peut modifier des lois à l'aide de preuves empiriques solides pour justifier le changement, en particulier lorsqu'elles sont combinées à suffisamment de pression de la part du public ${ }^{22}$. Même si ces études ont utilisé des paramètres applicables à des travailleurs de tout âge, ces résultats nous rappellent que des interventions de type descendant (topdown), par exemple des vérifications sécuritaires, peuvent constituer un moyen efficace de protéger les jeunes travailleurs.

\section{La voie à suivre : élaborer des paramètres appropriés pour analyser les programmes de prévention des blessures chez les jeunes travailleurs}

Une autre étude ne concordait pas avec notre cadre d'analyse mais s'est néanmoins révélée pertinente et importante. Wurzelbacher et Jin ont élaboré et testé un outil servant à prédire les résultats des indemnisations des accidentés du travail ${ }^{30}$. Ils ont défini des groupes de paramètres utilisables pour évaluer directement les interventions visant les blessures ou les maladies ("prévention primaire ») ainsi que les interventions visant à détecter les blessures ou les maladies avant qu'elles ne s'aggravent (" prévention secondaire ») ainsi que les interventions servant à réduire la durée du congé de maladie à la suite d'une blessure ou d'une maladie (« prévention tertiaire »). Ils ont regroupé les paramètres en fonction de leur valeur prédictive : d'une part les "paramètres principaux ", qui indiquent les risques ou les causes potentielles de blessures et sont donc utiles pour prédire de futures blessures, et d'autre part les "paramètres subséquents ", qui décrivent le passé mais ne sont pas nécessairement fiables pour prévoir l'avenir. Leur outil a été élaboré au moyen d'une analyse documentaire préliminaire servant à définir les principaux éléments des programmes de prévention des blessures. Des questionnaires détaillés ont ensuite été distribués aux entreprises participantes. Les questionnaires ont été créés à partir du programme de protection volontaire de l'OSHA et des programmes ergonomiques du NIOSH afin de saisir des éléments sur l'avant et l'après de la blessure. Des mesures des efforts de prévention de chaque entreprise, obtenues par autoévaluation, ont été combinées à des mesures des dommages antérieurs, telles que l'incidence des blessures et les demandes d'indemnisation. En combinant des paramètres principaux et des paramètres subséquents dans leur analyse, les chercheurs ont réussi à prouver l'efficacité de leur outil en prédisant les cas de demandes d'indemnisation sur la base des paramètres appliqués aux programmes de santé et de sécurité au travail des entreprises pariticipantes ${ }^{30}$. Même si les paramètres élaborés par Wurzelbacher et Jin concernent le secteur manufacturier et mettent l'accent sur les troubles musculosquelettiques, nous pensons que leur étude constitue un modèle intéressant pour les travaux futurs en matière d'élaboration de paramètres principaux valides spécifiques aux jeunes travailleurs dans d'autres contextes professionnels communs, sans compte leur application possible aux programmes de prévention des blessures chez les jeunes.

\section{Limites}

Cette analyse présente plusieurs limites. Premièrement, seuls des articles en anglais ont été analysés, ce qui a pu entraîner l'exclusion d'études pertinentes. Deuxièmement, à cause des limites de temps et d'argent, les stratégies de recherche manuelle n'ont pas fait partie de la méthodologie. Bien que la recherche comprenne des articles publiés au début de l'année 2015 , la limite de temps ne nous a pas permis d'effectuer des recherches plus récentes une fois la recherche affinée avec PICO (population, intervention, comparaison, résultat) effectuée. Afin de minimiser le risque de passer à côté d'un article utile, les critères de recherche ont été délibérément élargis afin d'obtenir le plus d'articles possible. Compte tenu du peu d'articles 
trouvés portant sur les programmes de prévention des blessures chez les jeunes travailleurs spécifiques aux lieux de travail réglementés par le gouvernement fédéral, cette stratégie a permis d'ouvrir la discussion en soulignant une lacune importante qui doit être comblée par de futurs travaux de recherche.

Finalement, l'objectif principal de cette analyse était d'exposer la portée des paramètres couramment utilisés pour évaluer les programmes de prévention des blessures chez les jeunes. L'évaluation de la qualité de chacune des études présentées dans l'analyse ne faisait donc pas partie de la méthodologie, et les résultats thématiques doivent donc être interprétés avec discernement ${ }^{31}$. Ces résultats visent à souligner les lacunes dans la discussion sur les accidents de travail chez les jeunes afin d'aider les experts à porter un regard critique sur les composantes de toute méthodologie d'évaluation appliquées à des programmes de prévention des blessures en milieu de travail. Cette étude se voulait un premier pas vers la détermination des priorités pour la recherche émergente sur l'évaluation de la prévention des blessures chez les jeunes travailleurs.

\section{Conclusion}

La littérature décrivant les paramètres d'évaluation des efforts de prévention des blessures chez les jeunes travailleurs en est encore à ses balbutiements. Les quelques études sélectionnées dans le cadre de notre examen ont fourni des renseignements sur la façon de déterminer la qualité d'une intervention, mais peu d'entre elles se sont avérées efficaces pour atteindre le but de tels efforts : réduire le nombre de blessures. Davantage d'études sont nécessaires pour identifier des paramètres efficaces aptes à établir des liens entre les programmes de prévention des blessures et la diminution concrète du taux de blessures. Il pourrait être utile d'intégrer aux évaluations futures de programmes le cadre élaboré par Wurzelbacher et Jin comme modèle de procédé pour élaborer des paramètres principaux valides aptes à prédire l'évolution des taux de blessures. En outre, d'autres travaux sont nécessaires pour explorer les raisons pour lesquelles certaines interventions en matière de sécurité fonctionnent alors que d'autres ne fonctionnent pas. La littérature actuelle suggère que la prévention des blessures chez les jeunes travailleurs devrait englober les catégories de l'éducation, de l'environnement et de l'application des règlements. La prochaine étape est d'élaborer des paramètres spécifiques aux jeunes aptes à prédire le taux de blessures au travail. Les chercheurs, les conseillers en matière de sécurité, les responsables des politiques et les planificateurs de programmes seront alors en mesure d'évaluer systématiquement les programmes en cours et de créer de nouveaux programmes fondés sur les meilleures données disponibles. Tant que des paramètres de qualité spécifiquement destinés aux jeunes ne sont pas systématiquement créés, un fossé va demeurer entre la recherche et les programmes fondés sur des données probantes pour prévenir les blessures chez les jeunes travailleurs.

\section{Remerciements}

Ce projet a été financé par le Programme de financement du travail d'Emploi et Développement social Canada.

Les auteurs remercient M. Takuro Ishikawa pour sa révision et ses suggestions concernant la section " méthodologie » de cette analyse.

\section{Conflits d'intérêts}

Les auteurs déclarent n'avoir aucun conflit d'intérêts.

\section{Contribution des auteurs et avis}

Tous les auteurs ont lu cette version de l'article et consenti à sa publication. IP et AM ont conçu l'étude et élaboré les protocoles de collecte de données et d'analyse de données. BPP a effectué l'acquisition des données. JS et BPP ont analysé les données. JS a rédigé le manuscrit. IP et AM ont fait la lecture critique et la révision du manuscrit.

Le contenu de cet article et les opinions qui y sont exprimées n'engagent que les auteurs et ne sont pas forcément représentatifs de la position du Gouvernement du Canada.

\section{Références}

1. Statistique Canada. Base de données CANSIM : Tableau 282-0018. Enquête sur la population active (EPA), estimations selon les heures effectivement travaillées, l'emploi principal ou tous les emplois, le sexe et le groupe d'âge, annuel [Internet]. Ottawa (Ont.) : Statistique Canada, [consultation le 7 octobre 2015]. En ligne à : http:// www5.statcan.gc.ca/cansim/a26?lang $=$ fra\&id $=2820018 \&$ retrLang $=$ fra\#F6
2. Statistique Canada. Base de données CANSIM : Tableau 282-0008. Enquête sur la population active (EPA), estimations selon le Système de classification des industries de l'Amérique $\mathrm{du}$ Nord (SCIAN), le sexe et le groupe d'âge, annuel (personnes x 1 000) (11) [Internet]. Ottawa (Ont.) : Statistique Canada, [consultation le 7 octobre 2015]. En ligne à : http:// www5.statcan.gc.ca/cansim/a26?lang $=$ fra\&id $=2820008 \&$ retrLang $=$ fra

3. Statistique Canada. Base de données CANSIM : Tableau 051-0001. Estimations de la population, selon le groupe d'âge et le sexe au 1er juillet, Canada, provinces et territoires, annuel (personnes sauf indication contraire) [Internet]. Ottawa (Ont.) : Statistique Canada, [consultation le 23 octobre 2015]. En ligne à : http:// www5.statcan.gc.ca/cansim/a26?lang $=$ fra\&retrLang $=$ fra\&id $=0510001$ \&pattern $=\&$ stByVal $=1 \& \mathrm{p} 1=1 \& \mathrm{p} 2=37$ $\&$ tabMode $=$ dataTable $\&$ csid $=$

4. Breslin FC, Smith P. Age-related differences in work injuries: a multivariate, population-based study. Am J Ind Med. 2005;48:50-56.

5. Association des commissions des accidents du travail du Canada. Tableau 3. Nombre d'accidents acceptés avec perte de temps, par âge et par province/territoire, 2011-2013 [Internet]. [consultation le 22 janvier 2016]. En ligne à : http://awcbc.org /fr/?page_id $=381$

6. Gouvernement du Canada. Règlement du Canada sur les normes du travail (C.R.C., ch. 986) employés de moins de 17 ans [Internet]. [consultation le 30 octobre 2015]. En ligne à : http:// laws-lois.justice.gc.ca/fra/reglements /C.R.C.\%2C_ch._986/page-2.html

7. Gouvernement du Canada. Industries et entreprises sous réglementation fédérale [Internet]. [consultation le 13 novembre 2015]. En ligne à : https://www.canada.ca/fr/emploi -developpement-social/programmes /equite-emploi/sous-reglementation .html 
8. Pratt B, Cheesman J, Breslin C, Do M. Accidents de travail impliquant des jeunes Canadiens : analyse de 22 années de surveillance des données recueillies à partir du Système canadien hospitalier d'information et de recherche en prévention des traumatismes. Promotion de la santé et prévention des maladies chroniques au Canada. 2016;36(5):101-112.

9. Smith PM, Saunders R, Lifshen M, et al. The development of a conceptual model and self-reported measure of occupational health and safety vulnerability. Accid Anal Prev. 2015;82: 234-243.

10. Rohlman DS, Parish M, Elliot DL, Montgomery D, Hanson G. Characterizing the needs of a young working population: making the case for total worker health in an emerging workforce. J Occup Environ Med. 2013; 55(12):S69-S72.

11. Breslin FC, Polzer J, MacEachen E, Morrongiello B, Shannon $\mathrm{H}$. Workplace injury or "part of the job"? towards a gendered understanding of injuries and complaints among young workers. Soc Sci Med. 2007;64(4):782-793.

12. Tucker S, Diekrager D, Turner N, Kelloway EK. Work-related injury underreporting among young workers: prevalence, gender differences, and explanations for underreporting. J Safety Res. 2014;50:67-73.

13. Groff P. The injury prevention spectrum and the 3 E's. In: Pike I, Richmond S, Rothman L, Macpherson A, editors. Canadian injury prevention resource. Toronto (Ont.) : Parachute; 2015. p. 51-55.

14. Burke MJ, Sarpy SA, Smith-Crowe K, Chan-Serafin S, Salvador RO, Islam G. Relative effectiveness of worker safety and health training methods. Am J Public Health. 2006;96(2): 315-324.

15. Banco L, Lapidus G, Monopoli J, Zavoski R. The Safe Teen Work Project: a study to reduce cutting injuries among young and inexperienced workers. Am J Ind Med. 1997; 31(5):619-622.
16. Lepping P, Lanka SV, Turner J, Stanaway SE, Krishna M. Percentage prevalence of patient and visitor violence against staff in high-risk UK medical wards. Clin Med. 2013;13(6): 543-546.

17. Mcdowell TW, Welcome DE, Warren $\mathrm{C}$, Xu XS, Dong RG. Assessment of hand-transmitted vibration exposure from motorized forks used for beachcleaning operations. Ann Occup Hyg. 2013;57(1):43-53.

18. Ulrey BL, Fathallah FA. Evaluation of a personal device in reducing the risk of low back disorders during stooped work. Work. 2012;41(Suppl. 1): 2381-2383.

19. Verbeek JH, Kateman E, Morata TC, Dreschler W, Mischke C. Interventions to prevent occupational noise induced hearing loss (Review). Cochrane Database Syst Rev. 2012;10(CD006396).

20. Adams A, Bochner S, Bilik L. The effectiveness of warning signs in hazardous work places: cognitive and social determinants. Appl Ergon. 1998; 29(4):247-254.

21. Salvatore AL, Chevrier J, Bradman A, et al. A community-based participatory worksite intervention to reduce pesticide exposures to farmworkers and their families. Am J Public Health. 2009;99(Suppl. 3):S578-S581.

22. Higgins DN, Casini VJ, Bost P, Johnson W, Rautiainen R. The Fatality Assessment and Control Evaluation program's role in the prevention of occupational fatalities. Inj Prev. 2001; 7(Suppl. I):i27-i33.

23. Haviland A, Burns R, Gray W, Ruder $\mathrm{T}$, Mendeloff J. What kinds of injuries do OSHA inspections prevent? J Safety Res. 2010;41(4):339-345.

24. Largo TW, Rosenman KD. Michigan work-related amputations, 2008. J Occup Environ Med. 2013;55(3): 280-285.

25. Kica J, Rosenman KD. Surveillance for work-related skull fractures in Michigan. J Safety Res. 2014;51: 49-56.
26. Rowlinson S, Jia YA. Application of the predicted heat strain model in development of localized, thresholdbased heat stress management guidelines for the construction industry. Ann Occup Hyg. 2013;58(3):1-14.

27. Arcury T, Quandt S, Russell G. Pesticide safety among farmworkers: perceived risk and perceived control as factors reflecting environmental justice. Environ Health Perspect. 2002;110(Suppl. 2):233-240.

28. Kahan E, Lemesh C, Pines A, Mehoudar O, Peretz C. Workers' rightto-know legislation: does it work? Occup Med. 1999;49(1):11-15.

29. Burt CDB, Williams S, Wallis D. New recruit safety expectations: relationships with trust and perceived job risk. Saf Sci. 2012;50(4):1079-1084.

30. Wurzelbacher S, Jin Y. A framework for evaluating OSH program effectiveness using leading and trailing metrics. J Safety Res. 2011;42(3):199-207.

31. Arskey H, O’Malley L. Scoping studies: towards a methodological framework. Int $\mathrm{J}$ Soc Res Methodol. 2005;8(1):19-32.

32. Chin P, DeLuca C, Poth C, Chadwick I, Hutchinson N, Munby H. Enabling youth to advocate for workplace safety. Saf Sci. 2010;48(5):570-579.

33. Ehlers JJ, Graydon PS. Noise-induced hearing loss in agriculture: creating partnerships to overcome barriers and educate the community on prevention. Noise Health. 2011;13(51): 142-146.

34. Linker D, Miller ME, Freeman KS, Burbacher T. Health and safety awareness for working teens. Fam Community Health. 2005;28(3): 225-238.

35. Schulte PA, Stephenson CM, Okun $\mathrm{AH}$, Palassis J, Biddle E. Integrating occupational safety and health information into vocational and technical education and other workforce preparation programs. Am J Public Health. 2005;95(3):404-411. 
36. Teran S, Strochlic R, Bush D, Baker R, Meyers J. Reaching teen farm workers with health and safety information: an evaluation of a high school ESL curriculum. J Agric Saf Health. 2008; $14(2): 147-162$.

37. Tucker S, Turner N. Waiting for safety: responses by young Canadian workers to unsafe work. J Safety Res. 2013;45:103-110. 